\section{Referral Bias and Other Perspectives on the HEDIS Measuring Stick for Quality of Care in Depression Treatment}

The National Committee for Quality Assurance (NCQA) is an independent not-for-profit organization based in Washington, DC, whose vision is to "to transform health care quality through measurement, transparency and accountability." Using the Health Plan Employer Data and Information Set (HEDIS), NCQA has determined how "more than 90 percent of the nation's managed care organizations" will collect, audit, and report their performance on a range of standardized quality measures. Many of the HEDIS measures are undisputedly useful in promoting population health, such as rate of beta-blocker use after myocardial infarctions or rate of cervical cancer screening. Other measures, such as antidepressant medication management (AMM), have less evidence supporting the goals promoted by NCQA.

Although the latest report on the State of Health Care Quality 2005 from NCQA appropriately describes and cites support for the need to treat depression, it does not cite supporting trials that validate the definitions chosen for their AMM measures. Specifically, the following definitions are used by HEDIS:

1. Effective Acute-Phase Treatment: the percentage of eligible members who were diagnosed with a new episode of depression, treated with antidepressant medication, and remained on antidepressant medication during the entire 12-week acute phase

2. Effective Continuation-Phase Treatment: the percentage of eligible members who were treated with antidepressant medication and remained on antidepressant medication for 6 months after diagnosis of a new episode of depression

3. Optimal Practitioner Contacts for Medication Management: the percentage of eligible members who received at least 3 follow-up office visits with a primary care physician or mental health provider in the 12-week acute-treatment phase after a new diagnosis of depression and prescription of antidepressant medication ${ }^{2}$

Why is it that NCQA can arbitrarily (as the self-appointed information source for managed care performance) define performance measures without citing studies showing improved outcomes when adhering to AMM recommendations? Yes, the current AMM measures sound reasonable; however, once incorporated into HEDIS, they become the performance standard, validated or not. AMM performance also becomes a ripe target for researchers and quality improvement initiatives.

In this issue of JMCP, Robinson et al. report on the percentage of 60,386 adult patients in the Marketscan (administrative) claims database who adhered to the AMM measures from January 2001 to September 2004. ${ }^{3}$ The primary outcomes were: $19 \%$ of patients achieved overall adherence to all 3 measures, while adherence rates for the 3 individual measures were 39\% for 3 or more practitioner contacts, $65 \%$ for acute-phase medication compliance, and $44 \%$ for continuation-phase compliance. Except for the optimal practitioner contact rate being higher than the national average ( $39 \%$ vs. 19\%), rates were very similar to the U.S. overall rates. ${ }^{2}$ Of note, when comparing the total health care costs with the payer, patients who had overall adherence to all 3 HEDIS measures had median total costs that were nearly 2 times higher over the 6-month follow-up period $(\$ 5,169$ vs. $\$ 2,734) .{ }^{3}$ What was the return on investment for the payer? Total costs for depression-related care in the adherent group were $\$ 1,922$ versus $\$ 677$ in the nonadherent group. ${ }^{3}$ If employers want to spend money wisely, what concrete patientoriented outcomes are being shaped by using the HEDIS AMM definitions? Until stronger linkage is established that shows that HEDIS depression management markers truly impact outcomes, the stream of studies joined by Robinson et al. should be redirected toward authenticating the AMM measuring stick, and not simply using it because it bears the HEDIS stamp of approval.

Besides AMM adherence rate determination from this administrative claims database, Robinson et al. also found that receipt of mental health specialty care was the only factor that was positively associated with greater adherence on all 4 measures (overall measure: odds ratio $[\mathrm{OR}]=3.895$; 95\% confidence interval $[\mathrm{CI}], 3.72-4.07) .{ }^{3}$ This take-home point was highlighted in the abstract, "(R)eceipt of mental health specialty care was the single factor most strongly associated with quality treatment by these measures." Taken at face value, this statement implies that patients are adversely affected if they do not receive care by mental health providers. The convenient AMM tool makes this claim possible, but was it applied appropriately?

An important confounder that affects database mining is referral bias to other specialties, such as mental health. Patients who are referred for mental health care are typically more difficult to treat, require greater attention, have more severe symptoms, and need greater duration treatment. In a study by Simon et al., managed care patients in Washington state who were started on new antidepressant medication were compared by treating specialty provider. ${ }^{4}$ They found, at baseline, that psychiatrists' patients reported slightly higher levels of functional impairment and greater prior use of specialty mental health care. During follow-up, psychiatrists' patients made more frequent follow-up visits, and the proportion making 3 or more visits in 90 days was 57\% versus 26\% for primary care physicians' patients; yet, despite these differences, the 2 groups showed similar rates of improvement in all measures of symptom severity and functioning. ${ }^{4}$

Another managed care study looking at outcomes for 358 patients receiving recommended depression treatment over 6 months showed that patients receiving less-than-recommended levels of antidepressant therapy for 90 days showed improvement in depression severity and health-related quality of life comparable with patients receiving minimum recommended 
treatment. In contrast to Robinson et al., the group receiving minimum recommended treatment had lower mean total medical costs over 6 months $(\$ 1,872 \pm \$ 140)$ compared with patients receiving less-than-recommended treatment $(\$ 2,622 \pm \$ 413$, $P=0.032){ }^{5}$

To appreciate the differences in depression populations between mental health and primary care, and to begin reflecting on the notion that universal application of the HEDIS AMM measures may not always be appropriate, consider a 12-month randomized controlled trial reported by Katon et al. of intensive follow-up versus usual care in 217 primary care patients. ${ }^{6}$ After stratifying into major and minor depression groups, they found that, for major depression (more likely to be referred for mental health care), the intervention group had greater adherence than the usual care controls to adequate dosage of antidepressant medication for 90 days or more $(75.5 \%$ vs. $50.0 \%, P<0.01)$, were more likely to rate the quality of the care they received for depression as good to excellent $(93.0 \%$ vs. $75.0 \% ; P<0.03)$ and were more likely to rate antidepressant medications as helping somewhat to helping a great deal $(88.1 \%$ vs. $63.3 \%, P<0.01)$. Seventy-four percent of intervention patients with major depression showed 50\% or more improvement on the Symptom Checklist-90 Depressive Symptom Scale compared with 43.8\% of controls $(P<0.01)$, and the intervention patients also demonstrated a significantly greater decrease in depression severity over time compared with controls $(P<0.004)$.

In contrast, for patients with minor depression (more likely to stay in primary care), Katon et al. found no significant differences between the intervention and control groups in the percentage of patients who were satisfied with the care they received for depression (94.4\% vs. $89.3 \%$ ), in the percentage who experienced a $50 \%$ or more decrease in depressive symptoms, or in the decrease of depressive symptoms over time.

Since database claims typically pull International Classification of Diseases, Ninth Revision (ICD-9) codes, appreciation for the variety and spectrum of depression severity is missing. Brief depressive symptoms or adjustment disorders with high rates of spontaneous resolution are commonly coded as depression not otherwise specified, (ICD-9 code 311) and do not require the intensity of treatment advocated by HEDIS. Although some patients in primary care may not receive the intensity of followup seen in mental health, this point does not require one to conclude that non-mental health specialty care is inferior. As noted above, with the limited research available, clinical outcomes are comparable in primary care even though followup rates and dosing levels may not meet the HEDIS AMM goals.
The roadmap for how depression treatment in primary care can improve further using a systematic chronic care model and primary care nurse managers has been thoughtfully illustrated by Solberg et al. and is worth reading by all those interested in quality improvement. ${ }^{7}$

Caution should be exercised with future application of HEDIS measures to ensure that outcomes are truly improved with NCQA definitions and that confounders such as referral bias are minimized when applying HEDIS to research efforts.

\section{Brian K. Crownover, MD, FAAFP, Lt. Col., MC, USAF, Associate Editor brian.crownover@eglin.af.mil bcrownover@amcp.org}

\section{DISCLOSURE}

The author is a board-certified family physician assigned to Eglin AFB Florida, where he serves as Family Medicine Residency program director, HQ Air Armament Center. The opinions and assertions contained herein are the private views of the author and are not to be construed as official or as reflecting the views of any organization, including the U.S. Air Force medical department or the U.S. Air Force. He discloses no potential bias or conflict of interest relating to this editorial.

\section{REFERENCES}

1. National Committee for Quality Assurance (NCQA). About NCQA Available at: http://www.ncqa.org/about/about.htm. Accessed December 20, 2005

2. National Committee for Quality Assurance (NCQA). HEDIS 2005. The state of health care quality, 2005. Industry trends and analysis. Available at: http://www.ncqa.org/Docs/SOHCQ_2005.pdf. Accessed December 20, 2005.

3. Robinson RL, Long SR, Chang S, et al. Higher costs and therapeutic factors associated with adherence to NCQA HEDIS Antidepressant Medication Management measures: analysis of administrative claims. J Manag Care Pharm. 2006;12(1):43-54

4. Simon GE, Von Korff M, Rutter CM, Peterson DA. Treatment process and outcomes for managed care patients receiving new antidepressant prescriptions from psychiatrists and primary care physicians. Arch Gen Psychiatry. 2001;58(4):395-401.

5. Revicki DA, Simon GE, Chan K, Katon W, Heiligenstein J. Depression, health-related quality of life, and medical cost outcomes of receiving recommended levels of antidepressant treatment. J Fam Pract. 1998;47(6):446-52.

6. Katon W, Von Korff M, Lin E, et al. Collaborative management to achieve treatment guidelines. Impact on depression in primary care. JAMA. 1995; 273(13):1026-3/1

7. Solberg LI, Trangle MA, Wineman AP. Follow-up and follow-through of depressed patients in primary care: the critical missing components of quality care. J Am Board Fam Pract. 2005;18(6):520-27. 